\title{
Géolinguistique
}

15 | 2015

La géographie linguistique au Brésil

\section{Les recherches géolinguistiques au Brésil : aperçu historique}

Geolinguistic Research in Brazil: A Historical Overview

Jacyra Andrade Mota

\section{(2) OpenEdition}

Journals

Édition électronique

URL : http://journals.openedition.org/geolinguistique/587

DOI : 10.4000/geolinguistique.587

ISSN : 2650-8176

Éditeur

UGA Éditions/Université Grenoble Alpes

\section{Édition imprimée}

Date de publication : 1 décembre 2015

Pagination : 7-24

ISBN : 978-2-84310-317-9

ISSN : 0761-9081

Référence électronique

Jacyra Andrade Mota, "Les recherches géolinguistiques au Brésil : aperçu historique », Géolinguistique [En ligne], 15 | 2015, mis en ligne le 15 février 2019, consulté le 10 novembre 2020. URL : http:// journals.openedition.org/geolinguistique/587 ; DOI : https://doi.org/10.4000/geolinguistique.587 


\title{
Les recherches géolinguistiques au Brésil : aperçu historique
}

\author{
Jacyra Andrade Mota \\ Universidade Federal da Bahia - CNPq (Brésil)
}

\section{Résumé}

Cet article traite du développement des recherches géolinguistiques au Brésil de 1826 à nos jours. En 1826, comme le remarque Nascentes (1952), on enregistre la première manifestation de nature dialectale sur le portugais du Brésil sous la plume de Domingos Borges de Barros, Visconde de Pedra Branca, dans son Introduction à l'Atlas ethnographique du Globe, œuvre organisée par le géographe vénitien Adrien Balbi. Les recherches dialectales sur le portugais du Brésil sont présentées en fonction de leur distribution dans les tranches indiquées par les périodisations proposées par Nascentes (1952), Cardoso et Ferreira (1994) et Cardoso et Mota (2006). Dans la troisième phase, de 1952 à 1996, on distingue l'élaboration d'atlas linguistiques régionaux dont le premier, l'Atlas Prévio dos Falares Baianos, de Nelson Rossi, qui a été publié en 1963 et, dans la quatrième, débutant en 1996, l'élaboration de l'Atlas Lingüístico do Brasil dont les premiers volumes sont parus en 2014. On termine avec des considérations sur la croissance significative des recherches géolinguistiques au Brésil.

\section{Mots-clés}

Les recherches géolinguistiques, la géolinguistique au Brésil, atlas linguistiques régionaux.

\section{Abstract}

This paper deals with the development of geo-linguistic research in Brazil from 1826 to nowadays. As noted by Nascentes (1952), the first manifestation of a dialectal nature regarding Portuguese in Brazil was registered 
in 1826 by Domingos Borges de Barros, Visconde de Pedra Branca, in his Introduction à l'Atlas ethnographique du Globe, a work organized by the Venetian geographer Adrien Balbi. The dialectal researches about Portuguese in Brazil are presented according to their distribution on the phases indicated by the periodization suggested by Nascentes (1952), Cardoso and Ferreira (1994) and Cardoso and Mota (2006). In the third phase (1952-1996) we highlight the construction of regional linguistic atlases, the first of which, the Atlas Prévio dos Falares Baianos, of Nelson Rossi authorship, was published in 1963, and in the fourth phase (1996present), the construction of the Atlas Linguístico do Brasil, whose first volumes were published in 2014. We conclude by bringing considerations on the significant growth of geo-linguistic research in Brazil.

\section{Keywords}

Geolinguistic research, Géolinguistics in Brazil, regional linguistic atlases.

\section{La géolinguistique au Brésil}

Au Brésil, la géolinguistique débute de façon systématique avec la réalisation des premières recherches dialectales à Bahia en 1957 sous la direction de Nelson Rossi dans le but d'élaborer l'Atlas Prévio dos Falares Baianos (APFB), atlas linguistique régional dont il est l'auteur, publié en 1963, avec la collaboration de Dinah Maria Isensée et Carlota Ferreira ${ }^{1}$ pour ne citer que les principaux auteurs. Selon Rossi : «A história do APFB a rigor remonta a 1955 - ano de nossa chegada a Bahia, já com a pretensão e esperança de fazer Dialectologia ${ }^{2} . »(1965$, p. 13)

La première recherche dialectale menée à Bahia a eu lieu en novembre 1957, comme le rapporte Andrade dans une communication présentée en 1958 au $1^{\text {er }}$ Congrès brésilien de dialectologie et ethnographie organisé à Porto Alegre :

O marco inicial das pesquisas dialetais que se vêm fazendo na Faculdade de Filosofia da Universidade da Bahia foi um exercício de campo realizado em Portão, no dia 1. de novembro de 1957. Tratava-se da primeira

1. Dinah Maria Isensée Callou est professeure à l'Université fédérale de Rio de Janeiro et Carlota da Silveira Ferreira à l'Université fédérale de Bahia.

2. «L'histoire de l'APFB remonte véritablement à 1955 , année où nous sommes arrivés à Bahia dans l'intention déjà et avec l'espoir de faire de la dialectologie.» 
aplicação de um questionário lingüístico experimental constante de 2.965 perguntas, elaborado com a colaboração dos alunos ${ }^{3}$. (1959, p. 3)

L'APFB est composé de 198 cartes linguistiques précédées de onze cartes introductives qui comprennent des données sur le parler bahianais selon la division dialectale proposée par Nascentes (1953), les localités composant son réseau de points et les enquêteurs de chaque enquête.

En ce qui concerne les informateurs, selon la méthodologie de la dialectologie monodimensionnelle inaugurée par Gilliéron dans l'Atlas linguistique de la France, ont été choisis 100 individus, de 39 à 69 ans, dont 83 analphabètes ou peu instruits. Toutefois, on ne constate pas, dans l'APFB, la prédominance de l'informateur de sexe masculin - le NORM (homme âgé de milieu rural, non mobile) ${ }^{4}$ cité par Chambers et Trudgill (1994, p. 57) ou le HARAS (homme, adulte, rural, analphabète et sédentaire) ${ }^{5}$ identifié par Zágari (2005, p. 52) - ; généralement, dans chaque localité, ont été interrogées deux personnes de sexe différent. Cette information se retrouve dans la liste des informateurs et peut être notée sur les cartes linguistiques.

Pour le choix du réseau de points, la proposition de Nascentes (1958) a été prise en considération dans Bases para a elaboração do Atlas Lingüístico do Brasil avec les ajustements jugés nécessaires.

Le questionnaire de 182 entrées, employé pour constituer le corpus de l'APFB, est extrait d'un questionnaire antérieur composé de plus de 3000 entrées appliqué lors d'enquêtes préliminaires dans quatre localités de province de l'État de Bahia.

Étant donné les conditions de l'époque, les réponses ont été transcrites in loco durant les enquêtes sans que soient employés des appareils d'enregistrement de la voix, qui ne seront disponibles que quelques années plus tard.

Quant aux cartes linguistiques, les transcriptions phonétiques, réalisées selon le système Lacerda Hammarström ${ }^{6}$, ont été dessinées à la main avec l'aide de normographes, comme l'explique Rossi :

3. «Le point de départ des recherches dialectales qui sont actuellement menées à la Faculté de philosophie de l'Université de Bahia fut une recherche sur le terrain, réalisée à Portão le $1^{\text {er }}$ novembre 1957. Il s'agissait de la première application d'un questionnaire linguistique expérimental de 2965 questions élaboré en collaboration avec les étudiants.»

4. De l'anglais : NORM (Non-mobile, Older, Rural Male).

5. Du portugais : HARAS (Homem, Adulto, Rurícola, Analfabeto e Sedentário).

6. Voir Lacerda et Hammarström (1953). 
Não é fácil, contando assim, dar uma idéia do que custava descobrir como utilizar os normógrafos disponíveis para chegar a um desenho aceitável dos sinais de transcrição que êles não continham. Foi necessário, muitas vezes, conjugar duas e até três réguas $25 A B, 35 A B$ e $45 A B$ (Rosenhain) [...] Mas nem sempre foi possível conseguir a pena exata que se utilizou inicialmente e, o que é pior, fomos forçados a substituir o primeiro desenhista por dois outros, de modo que nem assim se evitou de todo a irregularidade no desenho dos sinais ${ }^{7}$. (1965, p. 39-40)

La première édition étant épuisée, en 1998 a été préparée une édition sur CD-ROM à diffusion limitée, fruit de l'une des disciplines du programme d'études supérieures en lettres et linguistique à l'Université fédérale de Bahia ${ }^{8}$.

\subsection{Les origines de l'Atlas Prévio dos Falares Baianos (APFB) : 1950-1960}

Parmi les lointains antécédents à l'élaboration de l'APFB, on peut relever la première manifestation en faveur de la production d'un atlas linguistique du Brésil, consignée par le gouvernement brésilien au décret $30.643^{9} \mathrm{du}$ 20 mars 1952, confirmé par l'arrêté no 536 du 26 mai de la même année - fait que Cardoso et Ferreira (1994) considèrent être le point de départ d'une troisième phase dans l'histoire de la dialectologie brésilienne - et, étant donné les difficultés à élaborer, à cette époque-là, l'Atlas linguistique $d u$ Brésil, la proposition de produire un atlas linguistico-ethnographique du Brésil par régions, présentée par Silva Neto et Celso Cunha ${ }^{10}$ au III Colóquio Internacional de Estudos Luso-Brasileiros ( $3^{\mathrm{e}}$ Colloque international d'études luso-brésiliennes) en 1957 à Lisbonne.

7. «Il n'est pas facile, dans de telles circonstances, de donner un aperçu de la difficulté à découvrir comment utiliser les normographes disponibles pour arriver à un dessin acceptable des signes de transcription qu'ils n'offraient pas. Bien souvent il a fallu conjuguer deux, voire trois, règles $25 \mathrm{AB}, 35 \mathrm{AB}$ et $45 \mathrm{AB}$ (Rosenhain) [...] Mais il n'a pas toujours été possible d'obtenir la plume exacte employée au début et, pis encore, nous nous sommes vus contraints à remplacer le premier dessinateur par deux autres, de sorte que l'on n'a pu éviter complètement l'irrégularité dans le dessin des signes.»

8. Édition préparée par Américo Machado Filho, Sandra Cerqueira Prudêncio et Sílvia Santos da Silva, sous la direction de Jacyra Mota.

9. Le $3^{\text {e }}$ paragraphe du décret 30.643 déterminait, comme principale finalité du Centre de recherches de la Commission de philologie de la Casa de Rui Barbosa récemment créé, l'élaboration de l'Atlas linguistique du Brésil.

10. Voir Cunha et Silva Neto (1960). 
Exprimée auparavant par Silva Neto dans le Guia para Estudos dialectológicos dont la première édition parut en 1955, l'idée d'un atlas linguistico-ethnographique du Brésil par régions fut appuyée par Nascentes qui, dans Bases para a elaboração do atlas lingüístico do Brasil (vol. I, 1958), se prononçait ainsi :

Embora seja de toda vantagem um atlas feito ao mesmo tempo para todo o país, para que o fim não fique muito distanciado do princípio, os Estados Unidos, país vasto e rico e com excelentes estradas, entregou-se à elaboração de atlas regionais, para mais tarde juntá-los no atlas geral. Assim também devemos fazer em nosso país, que é também vasto e, ainda mais, pobre e sem fáceis vias de comunicação ${ }^{11}$. $(1958$, p. 7$)$

Dans cet ouvrage, Nascentes mentionne la division du pays en sept régions proposée par Silva Neto au $3^{\mathrm{e}}$ Colloque et suggère de démarrer «par des atlas de régions où ont déjà été effectués des sondages», citant «l'Amazonie, la Bahia et le Rio Grande do Sul» et ajoutant :

A título de sugestão, apresentamos um questionário típico, de caráter geral (cada região terá seu questionário suplementar especial), o que elaborámos em 1954 para o curso do professor Pop, o primeiro que se fez no Brasil, e uma lista dos pontos em que devem ser feitos os inquéritos ${ }^{12}$. (1958, p. 8)

Des antécédents plus proches se rapportent à l'action continue de plusieurs chercheurs, plus spécialement dans la deuxième moitié $\mathrm{du} \mathrm{xx}^{\mathrm{e}}$ siècle, et aux discussions sur le besoin d'élaborer l'Atlas linguistique du Brésil advenues lors de divers congrès, surtout de 1957 à 1959, période qui, comme l'observe Brandão, «é, sem dúvida, do mais alto significado na história da dialectologia brasileira ${ }^{13} \gg(1951$, p. 50).

Parmi les congrès les plus directement liés à la mise en œuvre de l'APFB on remarque, outre le $3^{\mathrm{e}}$ Colloque international d'études luso-brésiliennes

11. «Bien qu'il soit du plus grand intérêt qu'un atlas soit réalisé simultanément dans tout le pays pour que la fin ne soit pas trop éloignée du début, les États-Unis, vaste pays riche aux routes excellentes, se sont livrés à la préparation d'atlas régionaux rassemblés ultérieurement dans l'atlas général. Nous devons donc faire de même dans notre pays qui, lui aussi, est vaste et, de plus, pauvre et sans voies aisées de communication.»

12. «À titre de suggestion, nous présentons un questionnaire typique, de caractère général (chaque région aura son questionnaire complémentaire spécial), que nous avons élaboré en 1954 pour le cours du professeur Pop, le premier réalisé au Brésil, et une liste des points devant être mis en avant dans les enquêtes.»

13. «[...] est sans aucun doute de la plus haute importance pour l'histoire de la dialectologie brésilienne.» 
de 1957 déjà mentionné : (a) le I Congresso Brasileiro de Dialectologia e Etnografia tenu en 1958 à Porto Alegre; (b) le I Simpósio de Filologia Românica de 1958 aussi à Rio de Janeiro; (c) le IV Colóquio Internacional de Estudos Luso-Brasileiros de 1959 à Salvador.

$\mathrm{Au} 1^{\mathrm{er}}$ Congrès brésilien de dialectologie et ethnographie participèrent des chercheurs étrangers (du Portugal, d'Espagne, de France, d'Allemagne et d'Uruguay) et brésiliens, parmi lesquels Antenor Nascentes, Serafim Silva Neto, Celso Cunha, Nelson Rossi, Antônio Houaiss (secrétaire général), Sílvio Elia, Ismael de Lima Coutinho, Silveira Bueno, Cândido Jucá Filho et Heinrich Bunse. Rossi apporta à ce congrès les communications : Quadras populares no sertão baiano (Rossi, 1958), Cantigas de Rojão ${ }^{14}$ et, en collaboration avec des étudiants, Comércio de ervas medicinais na Feira de Água de Meninos. Andrade, dans la communication Pesquisas Dialectais na Universidade da Bahia, y présenta un rapport des activités de l'équipe bahianaise en 1958.

Parmi les communications apportées à ce congrès il convient de mentionner, en outre, pour son rapport au projet d'élaboration d'atlas régionaux, celle de Cândido Jucá Filho intitulée Questionário Lingüístico-etnográfico brasileiro qui, comme l'indique l'Introduction à Esboço de um Atlas Lingüístico de Minas Gerais (EALMG) :

[...] propunha uma grande rede nacional, controlada junto à antiga Faculdade de Filosofia e Letras do Rio Grande do Sul e integrada pelas demais Faculdades de Letras do país, Colégio Pedro II e Academia Brasileira de Filologia. Seu objetivo: o Atlas Lingüístico do Brasil. O projeto, primeiro que se levantou, nunca entrou, ao que se saiba, no terreno concreto das realizações ${ }^{15}$. (Ribeiro et al., 1977, p. 19)

$\mathrm{Du} 1^{\text {er }}$ Symposium de philologie romane on retient la proposition d'Aires da Mata Machado Filho qui recommande «la création d'une chaire de Linguistique Générale de caractère basique et introductif et l'établissement de l'étude autonome de la Dialectologie» (1970, p. 42), fait qui démontre l'importance que commencent à revêtir alors les études dans le domaine de la dialectologie.

14. Chansons de fêtes populaires interprétées sur un rythme rapide par des chanteurs du Nord-Est, souvent sous forme de défi.

15. «[...] proposait un grand réseau national contrôlé par la Faculté de philosophie et lettres du Rio Grande do Sul et intégré par les autres facultés de lettres du pays, le Collège Pedro II et l'Académie brésilienne de philologie. Son but : l'Atlas linguistique du Brésil. Le projet, le premier à être présenté, n'est jamais, à notre connaissance, entré sur le terrain concret des réalisations.» 
$\mathrm{Au} 44^{\mathrm{e}}$ Colloque international d'études luso-brésiliennes, l'équipe menée par Rossi présenta trois communications qui analysaient des données des enquêtes-sondage effectuées dans l'État de Bahia à Bom Despacho, en 1958, et à São José das Itapororocas, Tanquinho et São Vicente, en 1959. L'analyse de ces données et les discussions de Aspectos do léxico regional da Bahia (Rossi et al., 1959) allaient servir de base à l'élaboration de l'Extrato de Questionário appliqué dans tout l'État, d'octobre 1960 à avril 1961, pour constituer le corpus de l'APFB.

Outre celles déjà mentionnées, d'autres publications de l'époque contribuèrent à la préparation de la recherche débutante, telles que l'Introdução ao estudo da lingua portuguesa (Silva Neto, 1950) et Études dialectologiques au Brésil (Nascentes, 1952, 1953).

\subsection{Les projets de recherche dans le domaine de la géolinguistique au Brésil : 1963-1996}

La décision de produire, avant l'Atlas linguistique du Brésil, des atlas régionaux et la publication de l'APFB en 1963 vont contribuer, des années 1960 aux années 1990, à l'apparition de projets géolinguistiques qui, à une seule exception près, étaient aussi limités aux territoires des États.

Les atlas élaborés dans cette phase de la géolinguistique brésilienne suivent, pour la plupart, la méthodologie monodimensionnelle et ne portent leur attention que sur la dimension diatopique dans la ligne considérée aujourd'hui traditionnelle.

Il s'agit, énumérés ici dans l'ordre chronologique de leur date de parution bien qu'ils soient le fruit de projets entamés antérieurement, de : l'Esboço de um atlas lingüístico de Minas Gerais (EALMG), en 1977, résultat d'un projet de 1969; l'Atlas Lingüístico da Paraíba (ALPB), publié en 1984 à partir d'un projet démarré en 1979; 1'Atlas Lingüístico de Sergipe (ALS), inauguré en 1963, conclu en 1963 mais publié en 1987; l'Atlas Lingüístico do Paraná (ALPR), réalisé comme thèse de doctorat soutenue en 1990 et publié en deux volumes, l'un en 1994 et le second en 1996; l'Atlas Lingüístico-Etnográfico da Região Sul do Brasil (ALERS), dont les premiers volumes parus en 2002 provenaient d'un projet entrepris en 1980 ; l'Atlas Lingüístico de Mato Grosso do Sul (ALMS), élaboré en 2007 à partir d'un projet de 1994; et l'Atlas Lingüístico do Ceará (ALECE) en 2010, fruit d'un projet de 1978.

D'autres projets d'atlas restèrent inachevés ou inédits pour des raisons diverses. Il s'agit de l'Atlas Lingüístico do Estado de São Paulo (ALESP), l'Atlas Etnolingüístico dos pescadores do Estado do Rio de Janeiro (APERJ) et de l'Atlas Lingüístico e Etnográfico do Paraná. 


\subsubsection{Les atlas publiés}

En 1977, sous la plume de José Ribeiro, Mário Roberto Zágari, José Passini et Antônio Pereira Gaio, l'Esboço de um atlas lingüístico de Minas Gerais (EALMG) a été le premier atlas publié après l'APFB. Conçu en quatre volumes dont seul le premier est paru, il présente un ensemble de 73 cartes linguistiques dont 45 de type sémantico-lexical dans les domaines des phénomènes atmosphériques et des jeux d'enfants, et 28 contenant des isophones et des isolexiques identifiant des régions dialectales du Minas Gerais. Selon Zágari (2005, p. 59-60) ${ }^{16}$, le volume II devrait présenter les champs sémantiques absents du volume I, le volume III les traces de quimbundo ${ }^{17}$ dans les villes de Serro et Diamantina, et le quatrième une étude dialectométrique des données analysées.

Seuls les deux premiers des trois volumes prévus de l'Atlas Lingüístico da Paraíba (ALPB) de Maria do Socorro Silva de Aragão et Cleuza Bezerra de Menezes ont été publiés en 1984. Le premier contient 149 cartes linguistiques (lexicales et phonétiques) et le second l'analyse de phénomènes phonétiques et morphosyntaxiques documentés dans l'État de Paraíba ainsi qu'un glossaire des variantes cartographiées.

Selon Aragão, «l'Atlas Lingüístico da Paraíba fait partie d'un projet de recherche plus vaste, celui du relevé paradigmo-syntagmatique du lexique de Paraíba» (1998, p. 55) commencé en 1979.

Élaboré par une partie du groupe ayant participé à la construction de l'APFB (Carlota Ferreira, Jacyra Mota, Judith Freitas, Nadja Andrade, Suzana Cardoso, Vera Rollemberg et Nelson Rossi), l'Atlas Lingüístico de Sergipe (ALS) avait pour but de délimiter, vers le nord de l'État de Bahia, le domaine du parler bahianais, selon la proposition de Nascentes (1953). C'est pourquoi les quinze points choisis pour l'Atlas linguistique du Sergipe sont numérotés de 51 à 65 , à la suite des cinquante autres points intégrant la région de l'APFB.

L'ALS comprend 171 cartes linguistiques correspondant, en général, aux cartes de l'APFB et apporte 12 cartes conjointes Bahia-Sergipe mettant à profit des données non incluses dans l'APFB.

Du point de vue méthodologique, l'ALS inaugure la bi-dimensionnalité dans les atlas linguistiques brésiliens en incorporant systématiquement dans toutes les localités des informateurs des deux sexes, identifiés par les lettres A (les féminins) et B (les masculins).

16. Avec le décès de Zágari en 2010, il n’y a pas de nouvelles de la publication des volumes II, III et IV.

17. Langue du groupe Bantou parlée en Angola, apportée au Brésil par les esclaves africains. 
Le questionnaire employé pour constituer le corpus de l'ALS compte 686 questions, dont 181 tirées de l'Extrait de Questionnaire appliqué dans l'État de Bahia et 505 provenant d'enquêtes préliminaires appliquées en 1963 et 1964. En raison de l'étendue du questionnaire, la publication du premier volume de l'ALS en 1987 ne présente qu'un tiers du corpus disponible. Les deux autres tiers ont fait l'objet de plusieurs articles, communications à des congrès et d'autres travaux, parmi lesquels on remarque l'Atlas Linguiístico de Sergipe II, thèse de doctorat de Suzana Cardoso ${ }^{18}$ publiée en 2005, qui expose des données relatives au domaine de l'Homme (caractéristiques physiques et relations sociales) non cartographiées en 1987. Il se présente en deux volumes : l'un avec une introduction et l'autre avec 105 cartes linguistiques reprenant des informations lexico-sémantiques et phonético-phonologiques, et faisant ressortir graphiquement la variable diasexuelle. Dans le volume d'introduction, outre les informations méthodologiques, se trouvent des commentaires portant sur les cartes, comme prévu pour les atlas dits de deuxième génération, l'index des formes transcrites et l'index onomasiologique.

L'Atlas Lingüístico do Paraná (ALPR) de Vanderci de Andrade a tout d'abord été élaboré comme thèse de doctorat sous la direction de Rafael Eugênio Hoyos Andrade et présenté en 1990 à l'Université d'État Paulista (UNESP), campus d'Assis.

Il est constitué de deux volumes : le premier, publié en 1994, contient, outre les cartes introductives, 191 cartes linguistiques dont 92 lexicales, 70 phonétiques et 29 isoglossiques ou synthétiques, tandis que le second, Introduction, paru en 1996, apporte des informations méthodologiques. L'ALPR est aussi un atlas bidimensionnel qui allie la variable diasexuelle à la diatopique.

L'Atlas Lingüístico-Etnográfico da Região Sul do Brasil (ALERS), seul atlas brésilien à comprendre une région, soit les États de Paraná, Santa Catarina et Rio Grande do Sul, a débuté en 1980 sous la coordination de Walter Koch (UFRGS), avec la participation de Mário Klassmann (UFRGS), Cléo Altenhofen (UFPR), Oswaldo Furlan (UFSC), Hilda Vieira (UFSC), Felício Margotti (UFSC) et Basílio Agostini (PUC-Paraná).

Les deux premiers volumes (I. Introduction et II. Cartes phonétiques et morphosyntaxiques) publiés en 2002 furent dédiés à Heinrich Bunse ${ }^{19}$, chercheur qui «avait déjà entrepris, des années auparavant, des relevés préliminaires pour élaborer un atlas linguistique du Rio Grande do Sul»,

18. Soutenue en 2002 à l'Université fédérale de Rio de Janeiro.

19. Décédé en 1990. 
comme le font remarquer les organisateurs en parlant de l'origine, des objectifs et des fondements du projet ALERS (Koch, Altenhofen, Klassmann, 2011, p. 25).

En 2011 paraît une seconde édition rassemblant les volumes I et II, ainsi qu'un troisième volume, avec les cartes sémantico-lexicales, édité par Cléo Altenhofen et Mário Klassmann et dédié à Walter Koch ${ }^{20}$.

Le volume III présente 374 cartes sémantico-lexicales couvrant tous les domaines sémantiques du questionnaire. Il comprend, par ailleurs, l'introduction, des cartes auxiliaires, des tableaux de variantes pour des analyses linguistiques supplémentaires et des index.

L'Atlas Lingüístico de Mato Grosso do Sul (ALMS) a débuté en 1994 sous la coordination d'Albana Xavier Nogueira avec la participation de Maria José Toledo Gomes. En 1996, Nogueira en transmit la coordination à Dercir Pedro de Oliveira qui le publia en 2007. Ce dernier compta avec la collaboration d'Albana Xavier Nogueira, Aparecida Negri Isquerdo, Maria José Toledo Gomes, Ana Maria Pinto Pires de Oliveira, Maria Leda Pinto et Vitória Regina S. Ferreira.

Comme le notent Nogueira et Isquerdo (2005), le projet de réalisation de cet atlas est né de la suggestion et des encouragements de Pedro Caruso, ayant pour cela suivi de près l'orientation méthodologique de l'Atlas lingüístico do Estado de São Paulo avec, par ailleurs, l'emploi du même questionnaire. Par la suite, il subit l'influence des options méthodologiques adoptées par l'ALiB et commença à couvrir, outre la dimension diatopique, les dimensions diagénerationnelle et diasexuelle - avec l'inclusion de quatre informateurs par localité distincts quant au sexe et à la tranche d'âge - et la documentation des variantes phoniques au moyen de l'application d'un questionnaire phonético-phonologique (QFF).

L'ALMS contient 217 cartes linguistiques (57 phonétiques, 153 sémantico-lexicales et 7 morphosyntaxiques) en plus de la partie introductive et du chapitre final «Considerações sobre o léxico», élaboré par Regina S. Ferreira.

L'Atlas Lingüístico do Ceará (ALECE), élaboré sous la coordination de José Rogério Fontenele Bessa dès 1978, a été publié en 2010 par l'Université fédérale du Ceará. Il comprend deux volumes, l'un avec une introduction et l'autre avec des cartes linguistiques (108 lexicales et 132 phonétiques) qui présentent les données de 70 localités. Le questionnaire, publié en $1982^{21}$, réunit 306 questions à caractère sémantico-lexical et a été soumis à quatre

20. Décédé en 2008.

21. Voir Bessa et al. (1982). 
informateurs dans chaque localité, âgés de 30 à 60 ans, analphabètes ou ayant terminé l'éducation secondaire, employé aves les informateurs des deux sexes. Seules les réponses à 46 questions sélectionnées pour le critère de plus forte incidence de variation phonétique ont été cartographiées, comme le souligne l'auteur (Bessa, 2010, p. 7).

\subsubsection{Les projets qui ont été interrompus}

Le projet Atlas Lingüístico do Estado de São Paulo (ALESP) a été lancé en 1980 par Pedro Caruso (Universidade Estadual Paulista - Assis) et a, par la suite, bénéficié de la participation de Brian Head (alors à l'UNICAMP) avec l'archivage de copies d'enregistrements dans les deux universités. Avant le début des enregistrements, Caruso a réalisé une recherche-sondage par correspondance qui a atteint l'ensemble des 572 municipalités de l'État de São Paulo à cette époque-là. Après le sondage, 100 points furent sélectionnés, dont 58 correspondaient à ceux proposés par Nascentes. Les enquêtes (deux dans chaque localité) ont été menées à partir de l'application d'un questionnaire de 317 questions ${ }^{22}$ et, en 1998, selon l'auteur, elles furent réévaluées. Avec le décès de Caruso et l'éloignement de Brian $\mathrm{Head}^{23}$, le projet s'est trouvé interrompu sans que l'ALESP puisse être publié.

Le projet Atlas Etnolingüístico dos Pescadores do Estado do Rio de Janeiro (APERJ), démarré en 1985 à la Faculté de lettres de l'UFRJ, a été conçu et coordonné par Celso Cunha jusqu'à son décès en 1989, moment où la coordination en fut assumée par d'autres chercheurs, et fut interrompu en 1999 sans que l'atlas soit publié. Il convient toutefois de souligner que ce projet, étendu sur plus de dix ans, de type géosociolinguistique, intégré aux principes de la sociolinguistique, a fourni des données à d'innombrables travaux d'analyse, comme le remarque Brandão :

Essas pesquisas, em que fica nítida a interface Dialectologia/Sociolingüística, de um lado, representam uma significativa contribuição ao conhecimento da chamada variedade popular ou substandard do Português do Brasil, devendo-se observar que, no Estado do Rio de Janeiro, a quase totalidade dos trabalhos existentes sobre a fala de comunidades rurais e semiurbanizadas foi realizada por membros da Equipe APERJ com apoio no corpus coletado no final da década de 80 e inícios da de $90^{24}$. (2005, p. 362-363)

22. Voir Caruso (1983).

23. Pour l'Université du Minho, Portugal.

24. «Ces recherches, dans lesquelles l'interface dialectologie/sociolinguistique est claire, d'une part représentent une contribution importante à la connaissance de la variété dite populaire ou sous-standard du portugais brésilien et il convient de souligner 
Le projet Atlas Lingüístico e Etnográfico do Paraná aurait dû démarrer en 1973, mais n'a pas pu être mis en œuvre dans une perspective linguistique; il s'est vu limité à la recherche de caractère ethnographique développée par Júlio Alvar et Janine Alvar. Dans les pages d'introduction de l'ouvrage de ces chercheurs on peut lire :

Em 1973, devia ser iniciado o "Atlas Lingüístico e Etnográfico do Paraná". [...]. No mês de outubro de 1973, os autores encontravam-se em Curitiba, e o Projeto apresentava-se mais audacioso, pois que deveriam entrar outras matérias além da linguística e da etnografia. Entretanto, no momento do seu início, no dia 5 de novembro, não se havia chegado a um acordo sobre como realizar o trabalho, a fim de que realmente fosse um trabalho da equipe. Assim, de sua parte, os autores decidiram realizar um trabalho puramente etnográfico ${ }^{25}$. $(1979$, p. 15)

Les données ethnographiques documentées à Guaraqueçaba - municipalité choisie pour entamer les recherches au Paraná du fait de son isolement terrestre jusqu'en 1970 - apparaissent en 1979 dans une publication de l'Université fédérale du Paraná, Guaraqueçaba, mar e mato.

\subsection{Les projets de recherche dans le domaine de la géolinguistique de la fin du XX $X^{e}$ siècle à l'aube du XXI : 1996-2015}

Le début du projet Atlas Lingüístico do Brasil (ALiB) dans les dernières années du Xx siècle, en 1996 plus précisément, marque une nouvelle phase dans l'histoire de la dialectologie brésilienne, comme l'ont proposé Cardoso et Mota (2006), surtout vis-à-vis des recherches géolinguistiques, non seulement pour la stimulation d'innombrables autres projets dans ce domaine - certains élaborés sous forme de travaux d'études supérieures et orientés par des chercheurs participant au projet ALiB - mais aussi pour la méthodologie qui, dès lors, est adoptée pour les projets dans ce champ.

que, dans l'État de Rio de Janeiro, la presque totalité des travaux existants sur le parler des communautés rurales et semi-urbanisées a été menée par des membres de l'équipe APERJ s'appuyant sur le corpus recueilli à la fin des années 1980 et au début des années 1990.»

25. En 1973 devait démarrer l'Atlas linguistique et ethnographique du Paraná. [...] Au mois d'octobre 1973, les auteurs se trouvaient à Curitiba et le projet se présentait plus audacieux, car devraient y entrer d'autres matières en dehors de la linguistique et de l'ethnographie. Cependant, au moment de son démarrage, le 5 novembre, aucun accord quant à la façon de mener à bien le travail afin qu'il soit véritablement un travail d'équipe n'avait été convenu. Ainsi, de leur chef, les auteurs décidèrent de réaliser un travail purement ethnographique.» 
Les innovations méthodologiques de l'ALiB incluent l'emploi de questionnaires qui ne se limitent pas uniquement au lexique/sémantique et à l'adoption des principes de la dialectologie pluridimensionnelle contemporaine à laquelle se reporte Thun :

La nouvelle géolinguistique se caractérise par l'élargissement de son champ d'observation et par un travail en profondeur plus poussé. Elle passe de l'analyse de la superficie, constituée par la dimension diatopique, à celle de l'espace linguistique formé par la prise en considération de variables comme la dimension diastratique, diaphasique ou d'autres. D'autre part, la géolinguistique moderne se veut plus objective et représentative. Elle introduit la statistique dans le traitement des données. Elle fait de l'atlas silencieux, un atlas parlant. Elle n'emploie plus toute son énergie à la recherche du dialecte pur rural mais elle entre également dans les villes, elle analyse des langues régionales, focalise des situations de contact, questionne aussi des gens démographiquement mobiles. (2000, p. 408)

Le profil des informateurs adopté par l'ALiB est celui d'individus classés par sexe, tranche d'âge et, dans les capitales, niveau de scolarité, ce qui permet l'analyse d'autres variables, au-delà de la diatopie.

Les projets d'atlas entamés après 1996, certains déjà conclus, suivent les principes de la géolinguistique pluridimensionnelle contemporaine et, en général, la méthodologie de l'ALiB, avec les ajustements rendus nécessaires du fait de l'accroissement du réseau de points, du nombre d'informateurs ou de questions - surtout d'ordre lexico-sémantique, afin d'inclure des aspects caractéristiques du domaine étudié (manifestations folkloriques, produits locaux, etc.).

Ces projets peuvent être partagés en trois groupes : celui des atlas publiés, celui des atlas en cours, et celui des atlas présentés comme projets d'études supérieures (mémoires ou thèses).

Dans le premier groupe on citera l'Atlas Lingüístico Sonoro do Pará (ALiSPA) publié en 2004, premier atlas parlant brésilien, innovateur car il permet d'entendre, sur CD-ROM, la voix de l'informateur dans chacune des dix localités sélectionnées. Il a été réalisé sous la coordination d'Abdelhak Razky à partir d'un projet plus vaste de 1996 : l'Atlas Geo-sociolingüístico do Pará (ALiPA). Les enregistrements ont été faits en 2001 en employant la première version du questionnaire phonéticophonologique (QFF) de l'ALiB (cf. Comitê Nacional, 2001), avec des informateurs classés par tranche d'âge et sexe, toujours selon la méthodologie de l'ALiB.

Outre l'ALiPA, des projets d'atlas sont en chantier : (a) l'Atlas Lingüístico do Maranhão (ALiMA) en cours depuis 2000 sous la coordination de Conceição de Maria de Araújo Ramos (Université fédérale du Maranhão); 
(b) l'Atlas Lingüístico do Espirito Santo (ALES) démarré en 2006 sous la coordination de Catarina Vaz Rodrigues (Université fédérale de Espirito Santo) avec la participation de Vanderci de Andrade Aguilera; (c) l'Atlas EtnoLingüístico do Acre (ALAC), démarré en 1990, coordonné par Luísa Galvão Lessa; (d) l'Atlas Lingüístico de Rondônia (ALiRO) commencé en 2007 et coordonné par Iara Maria Teles (Université fédérale de Rondônia); (e) l'Atlas Lingüístico de Mato Grosso (ALiMAT) de 2009 sous la direction de José Leonildo Lima (Université fédérale du Mato Grosso); (f) l'Atlas Lingüístico do Rio Grande do Norte (ALiRN) débuté en 2009 sous la coordination de Maria das Neves Pereira (Université Potiguar) avec la participation de Maria do Socorro Aragão (Université fédérale de Paraíba); (g) l'Atlas Geo-sociolingüístico do Amapá (ALAP) débuté en 2010 sous la coordination d'Abdelhak Razky (Université fédérale du Pará) et la participation de Celeste Maria da Rocha Ribeiro (Université fédérale de l'Acre); (h) l'Atlas Lingüístico do Acre II (ALiAC II) coordonné par Lindinalva Messias do Nascimento Chaves (Université fédérale de l'Acre) démarré en 2014; (i) l'Atlas Lingüístico-Contatual das Minorias Alemãs na Bacia do Prata: cartografias do Hunsriqueano ${ }^{26}$ entre o Português e o Espanhol (ALMA-Cartographies) démarré en 2014 et coordonné par Cléo Altenhofen (Université fédérale de Rio Grande do Sul) et Harald Thun (Université de Kiel, Allemagne).

Quant aux atlas issus de projets d'études supérieures, en plus de l'Atlas Lingüístico de Sergipe II déjà mentionné pour sa relation au premier volume de l'Atlas linguistique de Sergipe, nombre ont été élaborés durant cette période, en général limités à un petit domaine, comme l'observe Romano :

No total, a Geolinguística brasileira conta com 35 atlas de pequeno domínio, dos quais 31 estão concluídos (10 teses de doutorado, 17 dissertações de mestrado, três monografias de especialização e um atlas resultado de projeto de Iniciação Científica) ${ }^{27}$. (2013, p. 235)

\section{Quelques mots de conclusion}

Après ses premiers pas en 1963 avec la publication de l'APFB, la géolinguistique brésilienne n'a vu s'accélérer son processus de cartographie du portugais dans la région brésilienne qu'à partir de la fin $\mathrm{du} \mathrm{xx}^{\mathrm{e}}$ siècle avec

26. Hunsriqueano : langue minoritaire parlée au sud du Brésil.

27. «Au total, la géolinguistique brésilienne compte 35 atlas de petit domaine, dont 31 sont conclus (10 thèses de doctorat, 17 mémoires de maîtrise, 3 monographies de spécialisation et un atlas résultat de projet d'Initiation scientifique.» Pour la liste de ces atlas, consulter Romano (2013, p. 232-233). 
la reprise du projet d'élaboration de l'Atlas Lingüístico do Brasil (ALiB) à l'occasion du séminaire "Chemins et perspectives pour la dialectologie au Brésil» tenu en 1996 à l'initiative du groupe de chercheurs de l'Institut de lettres de l'Université fédérale de Bahia sous la coordination de Suzana Alice Cardoso.

La preuve des difficultés auxquelles durent faire face les chercheurs de 1963 et 1996 dans ce champ de recherches est le nombre réduit d'atlas publiés jusqu'alors (cinq à peine), le temps écoulé entre le début des projets et les dates de publication des atlas, et la non-finalisation de certains projets.

L'intérêt pour la géolinguistique et la dialectologie au Brésil depuis 1996 est signalé par le nombre considérable de thèses et mémoires dans ce domaine, par l'élaboration d'atlas régionaux ou d'atlas de petit domaine et, depuis la constitution du corpus de l'ALiB, par l'analyse des données documentées pour définir des régions dialectales dans le portugais du Brésil.

Cette nouvelle phase est par ailleurs remarquable à plusieurs titres : pour l'élargissement du champ d'études qui ne se limite plus aux données phonético-phonologiques et lexico-sémantiques comme, en général, dans les atlas traditionnels, incorporant des données morphosyntaxiques, pragmatico-discursives, métalinguistiques, etc.; pour la présentation de données qui, dans les atlas actuels dits de seconde et troisième générations, sont accompagnées de commentaires linguistiques et de $\mathrm{CD}$ reproduisant la voix de l'informateur dans la localité où elle a été enregistrée; pour l'incorporation de nouveaux paramètres méthodologiques et l'abandon de la vision monodimensionnelle - monostratique, monogénérationnelle, monophasique, etc. - qui prédominait dans la géolinguistique brésilienne.

La publication des deux premiers volumes de l'Atlas Lingüístico do Brasil en 2014 (Cardoso et al., 2014) ainsi que la programmation des volumes suivants en cours, et la présence de disciplines liées aux études dialectologiques dans divers programmes universitaires supérieures brésiliens, prouvent aussi l'importance que prend aujourd'hui le domaine de recherches géolinguistiques dans ce pays.

\section{RÉFÉRENCES BIBLIOGRAPHIQUES}

Aguilera Vanderci de Andrade, 1990, Atlas Lingüístico do Paraná, thèse de doctorat, Universidade Estadual Paulista Júlio de Mesquita Filho, Assis.

Aguilera Vanderci de Andrade, 1994, Atlas Lingüístico do Paraná, Curitiba, Imprensa Oficial do Estado. 
Aguilera Vanderci de Andrade, 1996, Atlas Lingüístico do Paraná: apresentação, Londrina, EDUEL.

Altenhofen Cléo \& Klassmann Mário (éds), 2011, Atlas Lingüístico-Etnográfico da Região Sul do Brasil, II : Cartas semântico-lexicais, Porto Alegre, UFRGS ; Florianópolis, UFSC.

Alvar Júlio \& Alvar Janine, 1979, Guaraqueçaba, mar e mato, trad. de Cecília Maria Westphalen, Curitiba, Universidade Federal do Paraná, Setor de Ciências Humanas, Letras e Artes.

Andrade Nadja, 1958, Pesquisas Dialetais na Universidade da Bahia, I Congresso Brasileiro de Dialectologia e Etnografia, Porto Alegre, ms.

Aragão Maria do Socorro Silva de \& Menezes Cleuza Palmeira Bezerra de, 1984, Atlas Lingüístico da Paraíba, Brasilia, Universidade Federal da Paraíba, CNPq.

Bessa José Rogério Fontenele et al., 1982, Questionário do Atlas Lingüístico do Estado do Ceará, Fortaleza, Imprensa da Universidade Federal do Ceará.

Bessa José Rogério Fontenele (éd.), 2010, Atlas Lingüístico do Estado do Ceará, Fortaleza, Edições UFC.

Brandẽo Sílvia Figueiredo, 1991, A Geografia lingüística no Brasil, São Paulo, Ática.

BRANDÃo Sílvia Figueiredo, 2005, «A história e as contribuições de um projeto na linha geolingüística», dans V. d. A. Aguilera (éd.), A Geolingüística no Brasil: trilhas seguidas, caminhos a percorrer, Londrina, EDUEL, p. 357-370.

CArdoso Suzana Alice, 2002, Atlas Lingüístico de Sergipe II, thèse de doctorat, Faculdade de Letras, Universidade Federal do Rio de Janeiro, Rio de Janeiro.

CArdoso Suzana Alice, 2005, Atlas Lingüístico de Sergipe II, Salvador, EDUFBA.

CArdoso Suzana Alice et al., 2014, Atlas Lingüístico do Brasil, vol. 1 : Introdução, 212 p.; vol. 2 : Cartas linguísticas 1,368 p., Londrina, EDUEL.

Cardoso Suzana Alice \& Ferreira Carlota, 1994, A dialectologia no Brasil, São Paulo, Contexto.

Cardoso Suzana Alice \& Mota Jacyra, 2006, «Para uma nova divisão dos estudos dialetais brasileiros », dans J. Mota et S. Cardoso (éds), Documentos 2, Projeto Atlas Lingüístico do Brasil, Salvador, Quarteto, p. 15-26.

Caruso Pedro, 1983, Atlas Lingüístico do Estado de São Paulo: questionário, Assis, Instituto de Letras, História e Psicologia da UNESP, Prefeitura Municipal de Assis.

CAruso Pedro, 2005, «Metodologia da Pesquisa Dialetológica», dans V. d. A. Aguilera (éd.), A Geolingüística no Brasil: trilhas seguidas, caminhos a percorrer, Londrina, EDUEL, p. 371-380.

Chambers Jack \& Trudgill Peter, 1994, La dialectología, trad. de Carmen Morán González, Madrid, Visor Libros.

Comitê nacional do PRojeto ALiB, 2001, Atlas Lingüístico do Brasil: Questionários 2001, Londrina, EDUEL.

Cunha Celso Ferreira da \& Silva Neto Serafim da, 1960, «Atlas LingüísticoEtnográfico do Brasil», Actas do $3^{\circ}$. Colóquio Internacional de Estudos LusoBrasileiros (Lisboa, 1957), vol. 2, Lisboa, p. 405-412. 
Decreto $\mathrm{n}^{\circ} 30.643$, de 20 de Março de 1952. Institui o Centro de Pesquisas da Casa de Rui Barbosa e dispõe sobre o seu funcionamento. Diário Oficial da União, Rio de Janeiro, 22 mar. 1952. Seção 1.p. 4666. Disponible sur <www2. camara.leg.br/legin/fed/decret/1950-1959/decreto-30643-20-marco-1952 339719-publicacaooriginal-1-pe.html> [dernier accès le 9 aout 2013].

Ferreira Carlota et al., 1987, Atlas Linguiístico de Sergipe, Salvador, Instituto de Letras, Fundação Estadual de Cultura de Sergipe.

Gillieron Jules \& Edmont Edmond, 1902-1910, Atlas linguistique de la France, 35 fasc., Paris, Honoré Champion.

JuCÁ FILho Cândido, 1959, «Questionário Lingüístico-etnográfico brasileiro », I Congresso Brasileiro de Dialectologia e Etnografia (Porto Alegre, 1957), Separata da Revista de Portugal, série A língua portuguesa, vol. XXIV, p. 14-20. Disponible sur $<$ http://memoriaafrica.ua.pt/Catalog $>$ [dernier accès le 6 mars 2015].

Koch Walter, Klassman Mário \& Altenhofen Cléo (éds), 2002, Atlas Lingüístico-Etnográfico da Região Sul do Brasil, vol. I : Introdução, vol. II : Cartas fonéticas e morfossintáticas, Porto Alegre, UFRGS ; Florianópolis, UFSC; Curitiba, UFPR.

Koch Walter, Klassman Mário \& Altenhofen Cléo (éds), 2011, Atlas Lingüístico-Etnográfico da Região Sul do Brasil (ALERS), vol. I : Cartas fonéticas e morfossintáticas, $2^{\mathrm{e}}$ éd., Porto Alegre, UFRGS ; Florianópolis, UFSC, 512 p.

LACERDA Armando \& HAMmARSTRÖM Göran, 1953, «Transcrição fonética do português normal », Revista do Laboratório de Fonética Experimental, Faculdade de Letras da Universidade de Coimbra, vol. I, p. 119-135.

Machado Filho Aires da Mata, 1970, «Como se refletem na universidade brasileiraas atuais tendências da filologia românica», Anais do I Simpósio de Filologia Românica, Rio de Janeiro, MEC.

NASCENTES Antenor, 1952, «Études dialectologiques du Brésil», ORBIS, vol. 1, $\mathrm{n}^{\circ} 1, \mathrm{p} .181-184$.

NASCENTES Antenor, 1953, «Études dialectologiques du Brésil», ORBIS, vol. 2, $\mathrm{n}^{\circ} 2$, p. $438-444$.

NAscentes Antenor, 1953 [1922], O linguajar carioca, 2e éd., Rio de Janeiro, Simões.

Nascentes Antenor, 1958/1961, Bases para a elaboração do atlas lingüístico do Brasil, vol. 1/vol. 2, Rio de Janeiro, MEC, Casa de Rui Barbosa.

Nogueira Albana \& Isquerdo Aparecida, 2005, «Atlas Lingüístico de Mato Grosso do Sul: gênese e trajetória», dans V.d. A. Aguilera (éd.), A Geolingüística no Brasil: trilhas seguidas, caminhos a percorrer, Londrina, EDUEL, p. 229-250.

Oliveira Dercir Pedro (éd.), 2007, Atlas Lingüístico de Mato Grosso do Sul, Campo Grande, Ed. UFMS.

RAZKy Abdelhak (éd.), 2004, Atlas Linguiístico Sonoro do Pará (ALiSPA), Belém, CAPES/UFPA/UTM, éd. CD-ROM.

Ribeiro José et al., 1977, Esboço de um atlas lingüístico de Minas Gerais, vol. 1, Rio de Janeiro, Fundação Casa de Rui Barbosa, Universidade Federal de Juiz de Fora. 
Romano Valter Pereira, 2013, «Balanço crítico da Geolinguística brasileira e a proposição de uma divisão », Entretextos, Universidade Estadual de Londrina, vol. 13, p. 203-242.

Rossi Nelson, 1958, «Quadras populares no Sertão Baiano », Arquivos da Universidade da Bahia, Faculdade de Filosofia, Salvador, n 6, p. 85-134.

Rossi Nelson, 1963, Atlas Prévio dos Falares Baianos, Rio de Janeiro, Instituto Nacional do Livro, Ministério da Educação e Cultura.

Rossi Nelson, 1965, Atlas Prévio dos Falares Baianos. Introdução. Questionário Comentado. Elenco das respostas transcritas, Rio de Janeiro, Instituto Nacional do Livro, Ministério da Educação e Cultura.

Rossi Nelson et al., 1959, «Aspectos do léxico regional da Bahia», IV Colóquio Internacional de Estudos Luso-Brasileiros, Salvador, Universidade Federal da Bahia, datilografado.

Silva Neto Serafim da, 1950, Introdução ao estudo da língua portuguesa, São Paulo, Nacional.

Silva Neto Serafim da, 1957 [1955], Guia para estudos dialectológicos, 2e éd., Belém, Conselho Nacional de Pesquisas, Instituto Nacional de Pesquisas da Amazônia.

Thun Harald, 2000, «Atlas linguistique et variabilité - Introduction à la table ronde», Actes du XXII Congrès international de linguistique et de philologie romanes (Bruxelles, 1998), vol. III, Tübingen, Max Niemeyer, p. 407-409.

ZÁGARI Mário Roberto, 2005, «Os falares mineiros: esboço de um Atlas Lingüístico de Minas Gerais», dans V. d. A. Aguilera (éd.), A Geolingüística no Brasil: trilhas seguidas, caminhos a percorrer, Londrina, EDUEL, p. 45-72. 